\title{
THE COMMUTATOR SUBGROUPS OF THE ALTERNATING KNOT GROUPS
}

\author{
KUNIO MURASUGI
}

Abstract. The aim of this paper is to show that the commutator subgroup of the alternating knot group is the (proper or improper) free product of free groups with isomorphic subgroups amalgamated.

1. Main theorem. A surface $S \subset S^{3}$ is said to be algebraically unknotted if $\pi_{1}\left(S^{3}-S\right)$ is free. It was proved by Neuwirth [6] that any orientable surface of minimal genus spanned by a fibred knot is always algebraically unknotted. In this note, the following theorem will be proved.

THEOREM 1. Any alternating knot can span an algebraically unknotted orientable surface of minimal genus.

An immediate consequence of this theorem is the following.

COROLlaRY. The commutator subgroup of the knot group of an alternating knot can be written as

$$
\cdots \underset{F}{*} \underset{F}{*} F \underset{F}{*} F \underset{F}{*} \cdots,
$$

where $F$ is a free group of rank $2 g, g$ being the genus of the knot.

In $\S 3$, we construct a (not necessarily alternating) knot whose group has the commutator subgroup of the form (1).

Throughout this paper, we will use the same notation of [5].

2. Proof of Theorem 1. Let $k$ be an oriented knot of genus $g$ in a 3 -sphere $S^{3}$. Let $D$ be an orientable surface of minimal genus spanned by $k$. Therefore, the genus, $g(D)$, of $D$ is $g$. Let $V$ be a regular neighbourhood of $D$ in $S^{3} . D$, and hence $V$, can be deformed into a certain linear graph $L$ in $S^{3}$, where the Euler characteristic $\chi(L)$ of $L$ is given by $1-2 g$. Then the following lemma is obvious.

Lemma 1. If $L$ is a planar graph in $S^{3}$, then $\pi_{1}\left(S^{3}-L\right)$ is a free group of rank $1-\chi(L)$, and hence $D$ is algebraically unknotted.

Received by the editors July 6, 1970 .

AMS 1969 subject classifications. Primary 5520.

Key words and phrases. Algebraically unknotted surface, alternating knot, knot group, commutator subgroup, genus of knot, planar graph, primitive genus, knot diagram, Seifert circuit, primitive $s$-surface.

Copyright @ 1971, American Mathematical Society 
Now, let $K$ be a knot diagram of $k, p: S^{3} \rightarrow S^{2}$ a regular projection, and $p(k)=K$. For convenience, we select a "point of infinity" $\infty \in S^{3}-k$ and consider $S^{3}-\infty$ as a 3 -space $R^{3}$. Thus $p$ may be defined as $p(x, y, z)=(x, y)$ and $p(\infty)=\infty$. A knot diagram is said to be special if it does not contain Seifert circuits of the second type. By the primitive genus of a knot diagram $K$ is meant the integer $\frac{1}{2}[$ (number of $\alpha$-region)-(number of Seifert circuits of the second type) -1$]$, denoted by $g(K)$. We should note that the main theorem of $[3]$ is the following.

Proposition 1. The primitive genus of an alternating knot diagram of an alternating knot $k$ is equal to the genus of $k$.

Now the following lemma is the key to the proof of Theorem 1.

LemMA 2. Let $K$ be a knot diagram of $k$. Then there exists a knot $k^{\prime}$ of the same type as $k$ such that $p\left(k^{\prime}\right)=K^{\prime}$ is special and $g\left(K^{\prime}\right)=g(K)$.

Proof. If $K$ is already special, there is nothing to prove. Suppose that $K$ contains $n$ Seifert circuits of the second type. In the following, we call them simply S-circuits.

Now we wish to show that $k$ can be deformed to $k^{\prime}$ isotopically so that $K^{\prime}=p\left(k^{\prime}\right)$ contains at most $n-1$ S-circuits and has the same primitive genus as $K$.

Since an S-circuit is a simple closed curve in $S^{2}$, it divides $S^{2}$ into two domains. We take an innermost S-circuit, $C_{n}$ say, in the sense that one, $R_{1}$ say, of two domains $R_{1}, R_{2}$ in which $S^{2}$ is divided by $C_{n}$, does not contain other $\mathrm{S}$-circuits. Then without loss of generality we may assume that $R_{1}$ contains the origin $O$ of $R^{2}$ and any ray from $O$ meets $C_{n}$ at exactly one point. $C_{n}$ can be described as $(x(t), y(t))$, $0 \leqq t \leqq 1$. Choose a sufficiently small positive number $\lambda$ and let $C_{n}^{\prime}$ be the closed curve described by $((1+\lambda) x(t),(1+\lambda) y(t)), 0 \leqq t \leqq 1$. From the assumption on $C_{n}$, we see that $C_{n}^{\prime}$ is a simple closed curve and is "parallel" to $C_{n}$.

Let $P_{i}=\left(x\left(t_{i}\right), y\left(t_{i}\right)\right), i=1, \cdots, n$, be distinct double points on $C_{n}$ which do not belong to $C_{n}$. (For the definition, see [5, p. 390].) Then, in each small neighbourhood $U_{i}$ of $P_{i}$ in $S^{2}, C_{n}^{\prime}$ intersects with other S-circuits at two points $P_{i}^{\prime}, P_{i}^{\prime \prime}$. Then for small positive numbers $\epsilon_{i}, \eta_{i}, t_{i}-\epsilon_{i}$ and $t_{i}+\eta_{i}$ denote $t$-values of $P_{i}^{\prime}$ and $P_{i}^{\prime \prime}$, respectively. Define three points $A_{i}^{\prime}, B_{i}^{\prime}$, and $\hat{P}_{i}$ as follows. For a sufficiently small positive number $\epsilon$,

$$
\begin{aligned}
& A_{i}^{\prime}=\left(x\left(t_{i}-\epsilon_{i}-\epsilon\right), y\left(t_{i}-\epsilon_{i}-\epsilon\right)\right), \\
& B_{i}^{\prime}=\left(x\left(t_{i}+\eta_{i}+\epsilon\right), y\left(t_{i}+\eta_{i}+\epsilon\right)\right),
\end{aligned}
$$


and

$$
\hat{P}_{i}=\left((1-\lambda) x\left(t_{i}\right),(1-\lambda) y\left(t_{i}\right)\right) .
$$

Join two pairs of points $\left(A_{i}^{\prime}, \hat{P}_{i}\right)$ and $\left(B_{i}^{\prime}, \hat{P}_{i}\right)$ by line segments $a_{i}^{\prime}$ and $b_{i}^{\prime}$, respectively. Note that each of $a_{i}^{\prime}, b_{i}^{\prime}$ intersects with $C_{n}$ at only one point, but not with other S-circuits. Replacing each small arc $A_{i}^{\prime} B_{i}^{\prime}$ on $C_{n}^{\prime}$ by a broken line $a_{i}^{\prime} \cup b_{i}^{\prime}$, we will obtain a new simple closed curve $C_{n}^{\prime \prime}$.

Now, take two sufficiently close points $A, B$ on $C_{n}$, neither of which is a double point on $C_{n}$. By applying an obvious isotopy if necessary, we may assume that two points $p^{-1}(A) \cap k=a$ and $p^{-1}(B) \cap k=b$ are the highest points in $k$ and $k \subset S^{2} \times[-1,1]$.

Define a simple closed curve $\tilde{C}_{n}$ as $C_{n}^{\prime \prime} \times\{1\}$ in $S^{3}$. Let $A^{\prime}, B^{\prime}$ be two points on $C_{n}^{\prime}$ with the same $t$-value as $A, B$ and let $A_{0}, B_{0}$ be the points on $\widetilde{C}_{n}$ which are "above" $A^{\prime}, B^{\prime}$. Join two pairs of points $\left(a, A_{0}\right)$ and $\left(b, B_{0}\right)$ by line segments $\alpha, \beta$, respectively. Since $a, b$ are the highest points of $k$, the replacement of a small arc $a b$ of $k$ by $\alpha \cup \operatorname{arc} A_{0} B_{0} \cup \beta$ yields a new knot $k$ of the same type as $k$. Since $\widetilde{C}_{n}$ is above $k$, the further replacement of a small arc $A_{0} B_{0}$ of $k$ by the complement of arc $A_{0} B_{0}$ in $\tilde{C}_{n}$ again yields a new knot $k^{\prime}$ of the same type as $k$. $k^{\prime}$ thus obtained is the required knot. To prove this, we first note that every edge of $C_{n}$ is always on the boundary of some $\alpha$-region.

Since $C_{n}^{\prime \prime}$ is sufficiently close to $C_{n}$, each of those $\alpha$-regions, except two regions, which have the same edges of $C_{n}$ on their boundaries, is divided in to exactly one $\alpha$-region and one $\beta$-region. Therefore, in $K^{\prime}$, the original S-circuit $C_{n}$ disappears and other S-circuits remain untouched. Further, the exceptional regions are those which contain $A, B$ on their boundaries and these are amalgamated to make one big $\alpha$-region in $K^{\prime}$. Therefore, the primitive genus of $K$ and $K^{\prime}$ coincide. This completes the proof of Lemma 2.

Combining Proposition 1 and Lemma 2, we see that any alternating knot can be deformed isotopically into a knot $k^{\prime}$ so that $K^{\prime}=p\left(k^{\prime}\right)$ is special and $g\left(K^{\prime}\right)$ is the genus of $k$. Using this diagram $K^{\prime}$, we can construct an orientable surface $D$ according to Seifert's instruction given in [7]. Then the genus of $D$ is exactly the primitive genus of $K^{\prime}$, and hence, is the genus of $k$. That is to say, $D$ is an orientable surface of minimal genus. Finally, it is easy to see (for example, by considering the graph of knots [1], [2]) that $D$ is deformed into a certain planar graph in $S^{2}$.

This together with Lemma 1 imply Theorem 1.

3. A construction of algebraically unknotted surfaces. Although it is not known whether any knot can span an orientable algebraically 
unknotted surface of minimal genus, we can construct (not necessarily alternating) knots whose groups have the commutator subgroups of the form (1).

Take a finite connected planar graph $H$ with vertices $v_{1}, \cdots, v_{m}$ and edges $e_{1}, \cdots, e_{r}$. We assume that $H$ has no loops and the dual graph $\widetilde{H}$ of $H$ has even valency.

Now, replace each $v_{i}$ by a small disk $V_{i}$ and each $e_{j}$ connecting $v_{k}$ and $v_{l}$ by a band $E_{j}$ twisted once which attaches to two disks $V_{k}$ and $V_{l}$ as is shown in Figure 1 in [4]. The surface $S$ thus obtained is orientable, since $\widetilde{H}$ has even valency, and $S$ bounds a knot, or possibly a link. $S$ is called a generalized primitive s-surface associated with $H$ (although $S$ is not determined uniquely by $H$ ). Now, take two generalized primitive $s$-surfaces $S_{1}, S_{2}$ associated with $H_{1}, H_{2}$, and identify two disks, one from each of $S_{1}$ and $S_{2}$. For the details, refer to [4]. The resulting orientable surface is called a generalized $s$-surface. In general, by generalized s-surface is meant an orientable surface obtained from a finite number of generalized primitive $s$-surfaces by identifying them through disks in this manner. It is clear that a generalized $s$-surface is algebraically unknotted. Further, we can prove:

THEOREM 2. Let $k$ be the boundary knot of a generalized s-surface $S$ obtained from generalized primitive s-surfaces $S_{1}, \cdots, S_{n}$ in some manner. If each $S_{i}$ is of minimal genus which is spanned by a knot (or a link), then the knot group of $k$ has the commutator subgroup of the form (1).

Proof. Although $S$ is algebraically unknotted, we do not know whether $S$ is of minimal genus. However, to prove Theorem 2, it is sufficient to show that the homomorphism $\pi_{1}\left(S_{\#}\right) \rightarrow \pi_{1}\left(S^{3}-V\right)$ induced by inclusion is a monomorphism, where $S_{\sharp}$ denotes the "upper" boundary of $V$. However, it may be proved in almost the same manner used in [4], and therefore, will be omitted.

REMARK 1. It is easy to construct a generalized primitive $s$-surface satisfying the assumption of Theorem 2 . For example, if all bands are twisted once in the same direction, then the surface obtained satisfies the assumption.

REMARK 2. Every knot in Reidemeister's table spans an algebraically unknotted orientable surface of minimal genus.

\section{REFERENCES}

1. C. Bankwitz, Über die Torsionszahlen der alternierenden Knoten, Math. Ann. 103 (1930), 145-161.

2. S. Kinoshita and T. Yajima, On the graphs of knots, Osaka Math. J. 9 (1957), 155-163. MR 20 \#4845. 
3. K. Murasugi, On the genus of the alternating knot. II, J. Math. Soc. Japan 10 (1958), 235-248. MR 20 \#6103a.

4. - On a certain subgroup of the group of an alternating link, Amer. J. Math. 85 (1963), 544-550. MR $28 \# 609$.

5. - On a certain numerical invariant of link types, Trans. Amer. Math. Soc. 117 (1965), 387-422. MR 30 \#1506.

6. L. P. Neuwirth, The algebraic determination of genus of the knots, Amer. J. Math. 82 (1960), 791-798. MR 22 \#11397.

7. H. Seifert, Über des Geschlecht von Knoten, Math. Ann. 110 (1934), 571-592.

University of Toronto, Toronto, Canada 